\title{
BMJ Open Levofloxacin versus placebo for the treatment of latent tuberculosis among contacts of patients with multidrug- resistant tuberculosis (the VQUIN MDR trial): a protocol for a randomised controlled trial
}

Greg J Fox (D) ,,2 Cam Binh Nguyen, ${ }^{2}$ Thu Anh Nguyen, ${ }^{2}$ Phuong Thuy Tran, ${ }^{2}$ Ben J Marais, ${ }^{3,4}$ Steve M Graham, ${ }^{5,6}$ Binh Hoa Nguyen, ${ }^{7}$ Kavi Velen, ${ }^{2,3}$ David W Dowdy, ${ }^{8}$ Paul Mason, ${ }^{9}$ Warwick J Britton, ${ }^{3,10}$ Marcel A Behr, ${ }^{11,12}$ Andrea Benedetti, ${ }^{13}$ Dick Menzies, ${ }^{12}$ Viet Nhung Nguyen, ${ }^{7}$ Guy B Marks ${ }^{2,14}$

To cite: Fox GJ, Nguyen CB, Nguyen TA, et al. Levofloxacin versus placebo for the treatment of latent tuberculosis among contacts of patients with multidrug-resistant tuberculosis (the VQUIN MDR trial): a protocol for a randomised controlled trial. BMJ Open 2020;10:e033945. doi:10.1136/ bmjopen-2019-033945

- Prepublication history and additional material for this paper are available online. To view these files, please visit the journal online () .

Received 29 August 2019 Revised 22 0ctober 2019 Accepted 30 October 2019

D) Check for updates

(C) Author(s) (or their employer(s)) 2020. Re-use permitted under CC BY-NC. No commercial re-use. See rights and permissions. Published by BMJ.

For numbered affiliations see end of article.

Correspondence to

Dr Greg J Fox;

greg.fox@sydney.edu.au

\section{ABSTRACT}

Introduction Treatment of latent tuberculosis infection (LTBI) plays a substantial role in the prevention of drugsusceptible tuberculosis (TB). However, clinical trials to evaluate the efficacy of preventive therapy for presumed multidrug-resistant (MDR) LTBI are lacking. This trial aims to evaluate the efficacy of the antibiotic levofloxacin in preventing the development of active TB among latently infected contacts of index patients with MDR-TB.

Methods and analysis A double-blind placebocontrolled parallel group randomised controlled trial will be conducted in 10 provinces of Vietnam. Household contacts living with patients with bacteriologically confirmed rifampicin-resistant or MDR-TB will be eligible for recruitment if they have a positive tuberculin skin test or are known to be immunosuppressed, and do not have active TB. Participants will be randomised to receive either levofloxacin or placebo tablets once per day for 6 months. Screening for incident TB will be performed at 6 months intervals. The primary study outcome is the incidence of bacteriologically confirmed TB within 30 months after randomisation. Analysis will be by intention to treat, using Poisson regression.

Ethics Ethical approval from the University of Sydney Human Research Ethics Committee was obtained on 29 April 2015 (2014/929), and from the Vietnam Ministry of Health Institutional Review Board on 30 September 2015 (4040/QD-BYT).

Dissemination Findings of the study will be published in peer-reviewed publications and conference presentations. Trial registration number ACTRN12616000215426.

\section{INTRODUCTION}

Multidrug-resistant (MDR) tuberculosis (TB) is a major barrier to global TB control, affecting half a million people each year. ${ }^{1}$ MDR-TB has a substantially poorer prognosis than drug-susceptible TB (DS-TB),
Strengths and limitations of this study

This study is placebo-controlled, with participants, study staff and the trial statistician blinded to group allocation

- This study will offer treatment to adults and child contacts with latent tuberculosis (TB) infection, increasing its generalisability.

- Treatment is self-administered, meaning that adherence is likely to be reduced, but consistent with its application in TB control programmes.

- The sample size is dependent on the rate of incident TB, and hence if the incident TB rate is lower than expected, the power to detect an effect will be reduced.

and requires prolonged treatment with toxic and costly antibiotics to effect cure. ${ }^{2}$ Over 19 million people are estimated to have been latently infected with drug-resistant Mycobacterium tuberculosis. ${ }^{3}$ The treatment of latent $\mathrm{TB}$ infection (LTBI) is a top priority, as infected individuals provide a pool from which future TB cases will arise. ${ }^{4-6}$ Treatment of LTBI has a substantial role in the control of DS-TB and MDR-TB, and there is strong evidence for its effectiveness in reducing future TB disease risk. $^{7-9}$ In particular, effective treatment of contacts that have been exposed to patients with MDR-TB and have documented LTBI would prevent MDR-TB development and avoid the problems associated with its treatment. $^{1011}$

Observational studies have reported promising results using fluoroquinolone antibiotics as preventive therapy for drug-resistant 
LTBI. ${ }^{12-15}$ Consequently, recent WHO guidelines for treatment of LTBI recommend the use of fluoroquinolones to treat infected contacts of patients with MDR-TB. ${ }^{7}$ However, no randomised studies have evaluated the efficacy of LTBI treatment following household exposure to an infectious MDR-TB case.

Levofloxacin is a third-generation fluoroquinolone antibiotic that is a core drug within the standard regimen recommended to treat MDR-TB. ${ }^{16}$ The drug inhibits DNA gyrase, an enzyme that is essential for bacterial replication, and has both bactericidal and sterilising action against M. tuberculosis. ${ }^{17}$ Levofloxacin is well-tolerated, and has been widely used to treat other infectious diseases for up to 2 weeks. However, some studies of longer-term therapy have shown a significant incidence of musculoskeletal complications. ${ }^{18}$ Hence, while levofloxacin holds considerable promise for the treatment of drug-resistant LTBI, the efficacy and tolerability of prolonged fluoroquinolone therapy remain uncertain in the absence of randomised trial data.

The primary objective of this study will be to determine the efficacy of a 6-month regimen of levofloxacin in preventing the development of TB in infected household contacts of infectious MDR-TB patients. Secondary objectives are listed in online supplementary file 1 . These aims will be addressed by conducting a phase III clinical trial in TB-infected contacts of patients with MDR-TB recruited at clinics in Vietnam.

\section{METHODS AND ANALYSIS \\ Study design}

This is a double-blind parallel group randomised controlled trial. Participants in the intervention group will be given 6 months of self-administered oral levofloxacin once per day, while those in the control group will receive 6 months of self-administered placebo once per day. In both groups, participants will attend the clinic for assessment once monthly during the treatment phase. All participants will be followed for a total of 30 months after randomisation for the development of incident TB disease. The trial design is summarised in figure 1.

Intervention

Levofloxacin

Control

\begin{tabular}{|c|c|c|c|c|c|}
\hline $\begin{array}{c}\text { Screening } \\
\text { Phase }\end{array}$ & $\begin{array}{c}\text { Treatment } \\
\text { Phase }\end{array}$ & \multicolumn{3}{|c|}{$\begin{array}{l}\text { Follow-up } \\
\text { Phase }\end{array}$} & \\
\hline Months & $\begin{array}{lll}2 & 3 & 4\end{array}$ & 12 & 18 & 24 & 30 \\
\hline
\end{tabular}

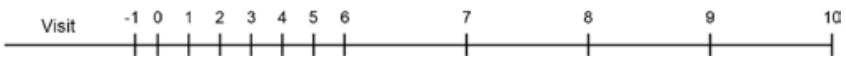

Figure 1 Summary of VQUIN MDR trial design.

\section{Setting}

This study will be conducted in Vietnam, a lower-middle income country of 96 million people in Southeast Asia. The country ranks among the high-prevalence countries for both TB and MDR-TB, and has a low population prevalence of HIV. ${ }^{1}$ Participants will be recruited in 10 provinces: Hanoi, Ho Chi Minh City, Can Tho, Da Nang, Quang Nam, Thanh Hoa, Khanh Hoa, An Giang, Tien Giang and Nam Dinh. These provinces include the provinces with the highest numbers of MDR-TB cases, comprising rural and urban sites throughout the northern, central and southern regions of Vietnam. Study sites will be clinics participating in the public Programmatic Management of Drug-Resistant TB Program, managed by the Vietnam National TB Program.

\section{Study population and eligibility criteria}

The study population will comprise household contacts of any age, living with a bacteriologically confirmed pulmonary MDR-TB index patient. Pulmonary MDR-TB is defined as the presence of sputum that is (a) acid-fast bacilli positive on smear microscopy, or positive for $M$. tuberculosis on culture or PCR using GeneXpert MTB/ RIF (Xpert) and (b) rifampicin resistant (RR) on PCR (Xpert) or resistant to both rifampicin and isoniazid on phenotypic or genotypic drug susceptibility testing (DST).

\section{Eligibility of index patients}

Consecutive index patients with bacteriologically confirmed pulmonary MDR-TB will be invited to enrol at participating clinics. If more than one index TB patient is identified within the same household, then the patient who has the earlier date of diagnosis will be considered as the index patient. A screening register will record the age and gender of consecutive eligible index patients who are approached to participate, enabling the representativeness of participants to be assessed. Index patients will be asked to invite their household contacts to attend the clinic for evaluation.

\section{Eligibility of household contacts}

Household contacts are defined as people living within the same household as the index case during the preceding 3 months, having shared the living space for at least one night per week. ${ }^{19}$ Household contacts be asked to provide written informed consent. Parents will provide consent for children under 15 years. Table 1 lists the detailed eligibility criteria for randomisation. Eligible household contacts require evidence of latent TB infection, without the presence of active disease.

Latent TB infection is defined as a tuberculin skin test (TST) of $10 \mathrm{~mm}$ or greater at the first reading, or TST conversion, defined as an increase of $6 \mathrm{~mm}$ or greater between the first and second reading, plus a second TST size of at least $10 \mathrm{~mm}$. A second TST will only be performed in contacts in whom the initial TST result is negative $(<10 \mathrm{~mm})$, and who have no evidence of active 
Table 1 Eligibility criteria for randomisation

\begin{tabular}{|c|c|}
\hline Inclusion criteria & Exclusion criteria \\
\hline $\begin{array}{l}\text { Aged } \geq 15 \text { years } \\
\text { TST positive (a size of } 10 \mathrm{~mm} \text { or greater at first reading); } \\
\text { OR } \\
\text { Any TST size if known to be HIV positive or severely } \\
\text { malnourished; } \\
\text { OR } \\
\text { New TST conversion on the second reading, defined as: } \\
\text { If the first test was }<5 \mathrm{~mm} \\
\text { a size of } 10 \mathrm{~mm} \text { or greater at second reading; OR } \\
\text { If the first test was } 5-9 \mathrm{~mm} \text { : } \\
\text { An increase of } 6 \mathrm{~mm} \text { or greater at the second reading }\end{array}$ & $\begin{array}{l}\text { A diagnosis of current active TB disease made during initial } \\
\text { assessment } \\
\text { Known to be pregnant* } \\
\text { Unable to take oral medication } \\
\text { Body weight }<3 \mathrm{~kg} \\
\text { Unwilling or unable to participate in follow-up for } 30 \text { months } \\
\text { Currently breast feeding } \\
\text { Known allergy to fluoroquinolone antibiotics, or history of } \\
\text { severe tendinopathy related to fluoroquinolones } \\
\text { Currently taking another medication reported to increase the } \\
\text { cardiac QT interval (eg, amiodarone, sotalol, disopyramide, } \\
\text { quinidine, procainamide, terfenadine) } \\
\text { Documented previous treatment for MDR-TB } \\
\text { Documented treatment with antibiotics that are active against } \\
\text { MDR-TB in the previous month (including fluoroquinolones) } \\
\text { Prior severe blistering reaction to tuberculin } \\
\text { End-stage liver failure (class Child-Pugh C) } \\
\text { Dialysis-dependent chronic kidney disease } \\
\text { A baseline liver function test (AST, ALT or ALP) more than } \\
\text { three times the upper limit of normal } \\
\text { Kidney tests show end-stage kidney disease (defined as an } \\
\text { EGFR }<20 \text { mL/min) } \\
\text { For adults aged } 15 \text { and above only: } \\
\text { Baseline ECG shows the QT segment (corrected for the R-R } \\
\text { interval) is }>450 \text { ms }\end{array}$ \\
\hline
\end{tabular}

*Women who are pregnant may be randomised after they have delivered and finished breast feeding. Women of childbearing age will be offered a urinary pregnancy test, though this will not be required as a condition of randomisation.

$\dagger$ ๆInitial assessment for active TB disease: At the time of enrolment, contacts aged $\geq 15$ years will be assessed for active disease, includingclinical assessment (including history and physical examination), plain chest radiograph and sputum microbiology (testing with Xpert, regardless of symptoms). This will be performed on: (a) all contacts aged 15 and above who are eligible to be randomized, and who are able to produce a sputum sample, and (b) all subjects aged 5 and above suspected to have TB

ALP, alkaline phosphatase; ALT, alanine transaminase; AST, aspartate aminotransferase; EGFR, estimated glomerular filtration rate; MDR-TB, multidrug-resistant tuberculosis; QT, corrected QT segment; TB, tuberculosis; TST, tuberculin skin test.

$\mathrm{TB}$ at the time of enrolment. For those requiring a second TST, the test will be performed after at least 8 weeks, to account for the long TST conversion window.

Baseline screening to exclude co-prevalent TB will comprise clinical assessment, chest radiography and sputum Xpert MTB/Rif (Xpert). In the presence of clinical, radiographic or Xpert-based evidence of possible TB, two additional expectorated sputum samples will be sent for both smear and liquid cultures. The decision to randomise participants will be delayed until the 8-week culture results are available. If the culture is negative, a repeat chest radiograph will be performed prior to randomisation to exclude incident TB.

Household contacts with previously known HIV infection, or other comorbidities that would suggest a compromised immune system, will be eligible for randomisation regardless of TST size-once active TB has been excluded. Pregnant women will be eligible for randomisation after they deliver and complete breast feeding.

\section{Recruitment of child contacts}

Child contacts are defined as those aged under 15 years of age. During an initial 12-month period, only adults aged 15 years and above will be randomised. If the prevalence of severe adverse events in adults is determined to be acceptably low by the Institutional Review Board of the Vietnam Ministry of Health, children will then be eligible to enrol. Until children become eligible for randomisation, those with LTBI will be offered serial chest radiograph and clinical surveillance for incident TB at 6 months intervals over 30 months of follow-up.

\section{Baseline assessment prior to randomisation}

Enrolled adult contacts will submit blood samples for the following tests prior to randomisation: complete blood count, liver function, kidney function tests, random blood sugar and a 12-lead ECG. Weight and height will be measured. Children who are being considered for randomisation will submit blood for baseline liver function tests (AST and ALT), full blood count and kidney function tests. 


\section{Randomisation}

The unit of randomisation will be individual household contacts. In households where two or more contacts are randomised within a 90-day period, all contacts in that household will be allocated to the same treatment group as the first contact in order to minimise a contamination effect. If additional contacts are enrolled 91 days or more after the first contact is enrolled, they will be randomised separately.

A stratified permuted block design will be used to perform randomisation, with varying block sizes (4-12 subjects). Block size will not be disclosed to study personnel, to minimise their likelihood of being able to predict the next randomisation assignment in the series. Separate randomisation lists will be generated by the trial statistician for each province to ensure balanced treatment group assignment. Randomisation will be performed through a web-based database.

\section{Blinding}

Participants and research staff will remain blinded to the identity of the study drug throughout the study. An indistinguishable placebo will be provided to those in the control group. A randomisation code will be provided to pharmacists dispensing the study drug at each site, allowing them to remain blinded to group allocation. The study drugs will be labelled and segregated such that the active drug and placebo are de-identified. Pharmacists, independent of the research staff, will perform dispensing of the study medication. The trial statistician will remain blinded to group allocation until the completion of the analysis. In case of a medical emergency, a clinician treating the study participant may submit a request for unblinding to a person independent of the study team. In this situation, group allocation will remain confidential and restricted to the managing clinician.

\section{Intervention}

The intervention comprises 180 doses of self-administered oral levofloxacin once per day, or an indistinguishable placebo. Tablets will be distributed once every 4 weeks by study staff, with a pill count performed at each visit.
The dosing range for levofloxacin will be $10-15 \mathrm{mg} /$ $\mathrm{kg} /$ day for adults, and $15-20 \mathrm{mg} / \mathrm{kg} /$ day for children. ${ }^{20}$ Two strengths of tablets will be used: 500 and $750 \mathrm{mg}$. The full dosing schema is shown in table 2. For children in weight band $10-24.9 \mathrm{~kg}$, tablets of 500 and $750 \mathrm{mg}$ will be split into two equal halves using a pill splitter. For children in weight band $3-9.9 \mathrm{~kg}$, tablets may be crushed and mixed with drinking water and the appropriate weight-based dose administered. If subjects have a serum creatinine above the normal range, then the creatinine clearance will be calculated. If the creatinine clearance is between 20 and $49.9 \mathrm{~mL} / \mathrm{min} / 1.73 \mathrm{~m}^{2}$, then the dose will be adjusted to half that of the normal adult dose. ${ }^{21}$

\section{Evaluation during the treatment period}

Household contacts will be assessed by study staff once in every month during the 6-month treatment period by research staff. At each visit, household contacts will be asked for symptoms of active TB disease and adverse events. At Months 1 and 2, blood will be collected from adult participants, with tests including liver and kidney function, and a random blood sugar in subjects known to be diabetic. In addition, an ECG will be performed at Month 1 visit to identify corrected QT segment ( $\mathrm{QT}_{c}$ ) prolongation among adult contacts, calculated according to the Fridericia formula. Children will have no routine blood tests or ECGs after the commencement of treatment, unless requested by the study clinician. Participants experiencing adverse events will be referred for a complete medical assessment by healthcare staff of the National TB Program. Adherence will be supported by phone contact every 2 weeks between scheduled monthly clinic visits.

\section{Evaluation during the period after treatment}

Participants will be asked to return for immediate review if they develop symptoms of TB during follow-up. Follow-up to assess for incident $\mathrm{TB}$ will be scheduled every 6 months, for a total of 30 months (ie, at 6, 12, 18, 24 and 30 months) after randomisation. At scheduled follow-up visits, contacts will be invited to have a symptom screen and chest radiograph, regardless of whether

Table 2 Weight bands for levofloxacin and placebo

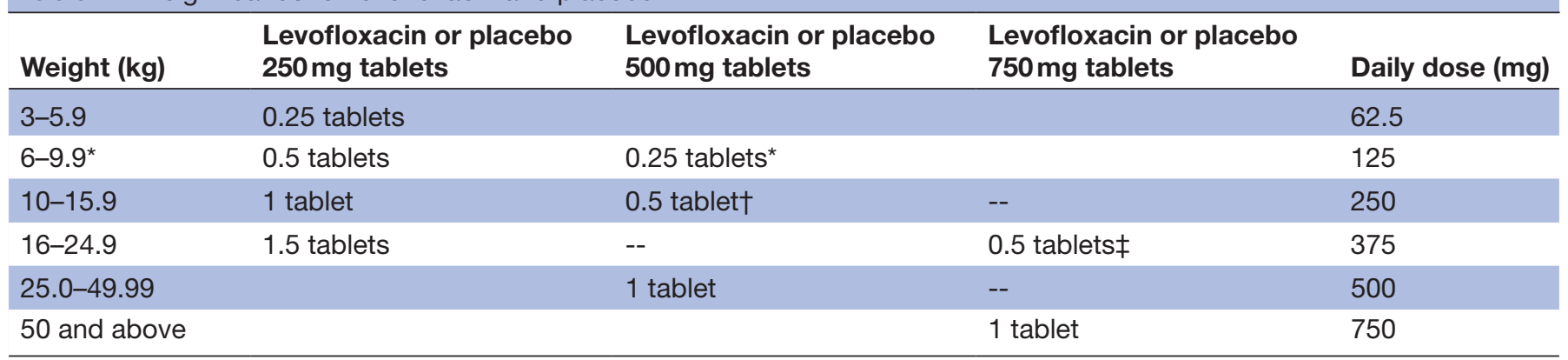

*If $250 \mathrm{mg}$ tablets are not available, $500 \mathrm{mg}$ tablets will be crushed and mixed with either (a) $10 \mathrm{~mL}$ drinking water, with $2.5 \mathrm{~mL}$ of the resulting mixture given to the child or (b) $5 \mathrm{~mL}$ of drinking water, with $1.25 \mathrm{~mL}$ of the resulting mixture given to the child.

†If $250 \mathrm{mg}$ tablets are not available, $500 \mathrm{mg}$ tablets will be divided into two parts, using a pill cutter.

†lf $250 \mathrm{mg}$ tablets are not available, $750 \mathrm{mg}$ tablets will be divided into two, using a pill cutter. 
symptoms are present. If contacts do not attend a scheduled appointment in person, they will be interviewed by telephone. In addition, at the conclusion of the 30-month follow-up, participants will be asked to produce a single sputum sample testing with Xpert MTB/RIF.

Additional follow-up interviews will be conducted by telephone at 9, 15, 21 and 27 months, to maintain a relationship with the study participant.

\section{Detection of incident TB}

Participants with symptoms of TB, or new radiographic abnormalities consistent with TB, will be asked to submit three sputum samples (the first for Xpert, and the remaining samples split for two additional smears and two liquid cultures). Confirmatory testing for M. tuberculosis will be performed when the culture is positive. Phenotypic DST will be performed on M. tuberculosis cultures. Study participants diagnosed with TB during the follow-up period will be treated with a standardised regimen for MDR-TB if RR is detected, or a standard regimen for DS-TB if RR is not detected. Trial medical advisors will provide guidance to local healthcare workers regarding the management of participants. M. tuberculosis bacilli isolated from index patients will be archived at central laboratories to enable subsequent molecular comparison with the isolates from contacts who develop active TB. Participants developing an adverse event related to the study drug will receive ongoing care through the National Tuberculosis Program facilities.

\section{Outcomes}

The primary outcome measure will be the incidence ratio of bacteriologically confirmed TB in the active levofloxacin group compared with the placebo control group. TB incidence will be measured from randomisation to end of follow-up, based on an intention-to-treat analysis.

Secondary outcome measures will include the following:

1. The incidence ratio of all forms of TB (bacteriologically confirmed or clinically probable) in the active levofloxacin group compared with the placebo control group.

2. The incidence ratio of all forms of TB (bacteriologically confirmed or clinically probable) in the active levofloxacin group compared with the placebo control group among subjects who completed therapy.

3. The incidence ratio of bacteriologically confirmed ТВ in the active levofloxacin group compared with the placebo control group; among contacts of MDR-TB patients with fluoroquinolone-susceptible disease, defined by phenotypic or genotypic testing of cultured isolates.

4. The proportion of participants in each group discontinuing treatment owing to adverse events.

5 . The proportion of participants in each group completing the trial therapy.

6. The ratio of the incidence of grade 3,4 or 5 adverse events in both groups occurring from the time of treat- ment commencement to 21 days after the last dose of treatment.

7. The ratio of death from any cause (except for violent or accidental causes) among the active levofloxacin group compared with the placebo control group.

8. The proportion of contacts with incident TB with acquired fluoroquinolone resistance in comparison with bacterial isolates of the index case, in the active levofloxacin group compared with the placebo control group.

9. The cost-effectiveness of levofloxacin therapy, compared with no treatment, expressed in the total cost per disability-adjusted life year averted, under programmatic conditions.

Completion of therapy is defined as taking at least 144 doses of preventive therapy (at least $80 \%$ of doses) within 270 days after commencing therapy. Complete definitions of 'bacteriologically confirmed' and 'clinically probable' pulmonary and extrapulmonary $\mathrm{TB}$ are included in online supplementary file 1 .

Participants will be censored from follow-up at the first date at which one of the following occurs: (a) 134 weeks passes since the date of randomisation, (b) when a study endpoint of bacteriologically confirmed or clinical TB is reached, (c) on the date of death or (d) on the last date of documented communication between the contact and any healthcare worker or study staff member prior to the close of the trial.

\section{Sample size}

Based on an earlier meta-analysis, we estimated that the cumulative incidence of TB among untreated MDR-TB contacts over a 2-year follow-up period will be 3.0\%. ${ }^{23}$ We estimate that levofloxacin will reduce incident TB by $70 \%$ in the treatment group, based on mid-range estimates of isoniazid efficacy for LTBI in DS-TB. ${ }^{24}$ The prevalence of fluoroquinolone resistance among MDR-TB patients in Vietnam was measured as $17 \%$, during the Fourth National Drug Resistance Survey conducted in 2011. ${ }^{25}$ Hence, we will increase the sample size to allow for a subgroup analysis of those contacts of patients with MDR-TB that is susceptible to fluoroquinolones. We will allow for a $10 \%$ dropout rate. We assume alpha $=0.05$ and the power to detect superiority is $80 \%$.

The sample size will be inflated to allow for clustering. The design effect $(1+(m-1) * \rho$, where $m$ is the size of the cluster and $\rho$ is the ICC (intraclass correlation coefficient)) is estimated as 1.04 at the district level with 21 index cases recruited, and 1.07 at the level of the household with 2.1 contacts per index case recruited. This is based on a previous study among household contacts in Vietnam ${ }^{26}$ where the ICCs at the district and household levels were estimated as 0.0018 and 0.0611 , respectively. Thus, the overall design effect is 1.1. The prevalence of LTBI in household contacts of MDR-TB patients is expected to be at least $60 \% .{ }^{23}$ Hence, the target sample size is 2006 randomised contacts. ${ }^{27}$ Based on an expected prevalence of infection of $60 \%$ among household 
contacts, we expect to screen a total of approximately 3344 contacts.

\section{Trial governance}

The implementation of the trial will be overseen by a Trial Steering Committee. An Independent Data Monitoring Committee (IDMC) will be established to protect the validity and scientific credibility of the trial, and will be independent of the funding agency and study sponsor. The IDMC will review reported adverse events and monitor participant safety.

An Endpoint Review Panel (ERP), including adult and paediatric TB physicians, will adjudicate the diagnosis of all contacts with suspected or confirmed TB. The ERP will also classify and grade adverse events. The panel will consist of members who are distinct from, and independent of, the Trial Steering Committee, and will adjudicate the primary endpoint on the basis of documented clinical information about all contacts with suspected $\mathrm{TB}$, blinded to the drug administered.

\section{Ethics and dissemination}

\section{Ethical issues}

Adults will provide written informed consent. A sample Participant Consent Form for adults is located in online supplementary file 2 . Children aged under 15 years will require written parental consent, and will be asked to give verbal assent. Research participants may voluntarily withdraw from the study for any reason. Written consent for ancillary studies, such as studies of levofloxacin pharmacokinetics and the effect of levofloxacin on the microbiome, will be provided separately. All records will remain confidential throughout the study. Study data will be stored on password-protected electronic devices. Written records will be stored securely in the District clinics and Provincial hospitals, at the National Lung Hospital or in other secure research facilities. Incentive payments of approximately US $\$ 5$ will be made to participants to compensate them for their time and travel expenses required to attend each visit.

\section{Dissemination}

Study findings will be presented at international conferences and submitted for publication in an international peer-reviewed journal. The full protocol and statistical code for the primary analyses will be included as appendices accompanying the publication that presents the main trial results. Data from the study will be de-identified and available for subsequent analysis based on request to the investigators. Authorship will be determined based on the recommendations of the International Committee of Medical Journal Editors. ${ }^{28}$ The SPIRIT Checklist for this protocol is located in online supplementary file 3 .

\section{Patient and public involvement}

The trial was informed by patients' need for an effective preventive therapy for MDR LTBI, since randomised trial data are lacking. Patients were not involved in the design of the study. A summary of the main trial results will be disseminated to study participants, via email or post, after the completion of the study.

\section{Data analysis}

The proportion of participants reaching study endpoints will be expressed per 100 person-years of follow-up. The efficacy of levofloxacin will be assessed by comparing the cumulative incidence of bacteriologically confirmed TB among those randomised to the intervention group with the cumulative incidence among those randomised to the control group. The primary efficacy analysis will be by intention to treat. In this primary analysis, there will be no adjustment for baseline characteristics. We will use a marginal Poisson regression model estimated via a generalised estimating equation (GEE) to test the effect of treatment group allocation on the incidence ratio of TB. Follow-up time (the time from enrolment to censoring) will be included as an offset to account for differing lengths of follow-up depending on date of study entry. Models estimated via GEE will adjust inference (eg, CIs and p values) to account for correlation between subjects clustered by district and/or household and are robust to mis-specification of the correlation structure. We will use an exchangeable correlation structure and the empirical standard errors. On account of the expected low frequency of events in a single district or household, in sensitivity analyses we will ignore clustering.

A secondary modified intention-to-treat analyses will exclude household contacts of index patients who are incorrectly classified (eg, those in whom the index patient was positive for RR on GeneXpert, but phenotypically susceptible to rifampicin and/or isoniazid), and contacts who did not commence therapy on account of early withdrawal prior to treatment.

To estimate the incidence of acquired drug resistance among contacts taking therapy, we will use a Poisson marginal model estimated via GEE with outcome being drug-resistant TB, and using follow-up time as an offset. In other analyses, we will investigate predictors of acquired drug resistance by comparing subjects who develop drug-resistant TB with those who develop TB without acquired drug resistance via a marginal logistic regression model.

\section{Cost-effectiveness analyses}

Costs for diagnosis and treatment will be estimated using questionnaires of staff, patients and contacts at provincial and district level healthcare facilities. Costs to be measured will include out-of-pocket expenses, overhead and facilities, costs of drugs and other consumables, human resource costs for the health system and lost wages for patients. Costs of the intervention will be assessed using a micro-costing approach, in which all costs are individually enumerated and converted to unit costs. We will estimate costs from both the health system and the patient perspective. 


\section{Data management and quality control}

Data will be recorded on paper forms at District clinics, and entered into a custom-designed online database by study staff. Chest radiographs will be read independently by two readers, including a member of the clinic and a study medical advisor. Where discrepancies are identified between the readings, a third reader will adjudicate between the first two readings. Quality control of laboratory testing will include routine structured quarterly monitoring visits by staff from the National Lung Hospital. Quality control processes are detailed in online supplementary file 1 . The investigators will have access to the final trial data set. Microbiological isolates collected from study participants, and blood samples collected for biomarker testing, will be stored for use in subsequent ancillary studies.

\section{Evaluation and monitoring}

Senior research staff will conduct internal monitoring and evaluation in each province at least every 6 months to evaluate study performance. Monitoring visits will include checking participant consent forms, source data verification, protocol compliance and data quality. An external monitoring team will be appointed by the Trial Steering Committee to provide periodic external review of the conduct of the trial, in order to make recommendations to strengthen compliance with regulations.

\section{DISCUSSION}

Treatment of LTBI promises to reduce incident TB among close contacts of patients with pulmonary MDR$\mathrm{TB}$, thereby limiting transmission and reducing health system costs. Treatment trials among persons with DS-TB have demonstrated a $60 \%-90 \%$ reduction in incident $\mathrm{TB},{ }^{29}$ and form the basis for national and international guidelines recommending their adoption among close contacts. ${ }^{7}$ However, existing evidence for LTBI treatment following MDR-TB exposure is limited.

Observational studies, performed in a range of highprevalence countries, indicate that fluoroquinolones hold considerable promise for preventing drug-resistant TB. ${ }^{12}$ A prospective cohort study in Micronesia showed that among 110 contacts of MDR-TB patients, given a 12-month directly observed course of a fluoroquinolone, with or without additional companion drugs (ethambutol and/or ethionamide), no cases of TB were identified during 12 months of follow-up. ${ }^{14}$ A South African study of 184 child contacts of MDR-TB patients also found very low rates of incident $\mathrm{TB}$ disease using a 6-month preventive therapy regimen that included a fluoroquinolone (ofloxacin). ${ }^{13}$ Both studies reported low rates of adverse events and good tolerability. However, these studies lacked a control group and had mean follow-up periods of under 2 years. In contrast, the present study will evaluate the effect of levofloxacin on microbiologically confirmed disease over a 30 -month period in a placebo-controlled trial.
If levofloxacin is effective in reducing the incidence of TB in this population, further evaluation will determine its cost-effectiveness and acceptability to patients. Evaluating the acquisition of fluoroquinolone resistance and the adverse consequences of long-term fluoroquinolone use on the human microbiome will also be important. Additional companion studies are planned to evaluate these concerns.

\section{CONCLUSION}

The VQUIN MDR Trial addresses the call for randomised trials to assess prevention strategies among close contacts of infectious MDR-TB cases, in recent WHO Consolidated Guidelines for the programmatic management of LTBI. ${ }^{7}$ The evidence arising from this study will be relevant to the management of infected contacts of patients with pulmonary MDR-TB in other settings, enabling national TB programmes to develop evidence-based responses to the growing threat posed by transmitted drug-resistant TB.

\section{CONTACT FOR PUBLIC AND SCIENTIFIC QUERIES}

The contact for scientific and public queries is the Principal Investigator, Dr Greg J. Fox (greg.fox@sydney.edu. au). Rm 5216, Level 2 Medical Foundation Building K25, 92-94 Parramatta Road, The University of Sydney, NSW AUSTRALIA 2006.

\section{TRIAL STATUS}

Recruitment completed.

\section{Author affiliations}

${ }^{1}$ Central Clinical School, The University of Sydney Faculty of Medicine and Health, Sydney, New South Wales, Australia

${ }^{2}$ Woolcock Institute of Medical Research, Glebe, New South Wales, Australia ${ }^{3}$ The University of Sydney Faculty of Medicine and Health, Sydney, New South Wales, Australia

${ }^{4}$ The University of Sydney Marie Bashir Institute for Infectious Diseases and Biosecurity, Sydney, New South Wales, Australia

${ }^{5}$ Murdoch Childrens Research Institute, Parkville, Victoria, Australia

${ }^{6}$ Department of Paediatrics, The University of Melbourne, Melbourne, Victoria, Australia

${ }^{7}$ The National Lung Hospital, Hanoi, Vietnam

${ }^{8}$ Department of Epidemiology, John Hopkins Bloomberg, Baltimore, Maryland, USA

${ }^{9}$ Taronga Institute of Science and Learning, Taronga Conservation Society, Sydney, New South Wales, Australia

${ }^{10}$ Tuberculosis Research Program, The Centenary Institute of Cancer Medicine and Cell Biology, Sydney, New South Wales, Australia

${ }^{11}$ Department of Medicine, McGill University, Montreal, Quebec, Canada

${ }^{12}$ McGill International Tuberculosis Centre, McGill University, Montreal, Quebec, Canada

${ }^{13}$ Departments of Medicine and of Epidemiology, Biostatistics \& Occupational Health, McGill University, Montreal, Quebec, Canada

${ }^{14}$ South Western Sydney Clinical School, University of New South Wales, Sydney, New South Wales, Australia

Contributors GJF wrote the proposal for this study that was awarded funding. GJF, CBN, TAN, BM, SG, BHN, DWD, WJB, MB, AB, DM, VNN and GBM made important intellectual contributions to the study protocol. GJF, CBN, TAN, BM, SG, BHN, DWD, WJB, MB, AB, DM, VNN and GBM contributed to the multiple revisions of this study 
protocol. GJF and AB performed statistical calculations for sample size and power. GJF wrote the first draft of the manuscript. CBN, TAN, BM, SG, BHN, PTT, KV, DWD, WJB, MB, AB, PM, DM, VNN and GBM contributed substantially to manuscript versions. All authors revised and approved the final manuscript. The funding agency played no part in any aspect of the study, nor the decision to submit this manuscript for publication.

Funding This work is supported by the Australian National Health and Medical Research Council (NHMRC) grant application number 1081443. GJF was supported by a NHMRC CJ Martin Fellowship (APP 1054107) and NHMRC Career Development Fellowship (APP 1148372). The trial sponsor is the Woolcock Institute of Medical Research, 431 Glebe Point Road, Glebe, NSW Australia, 2037.

Competing interests None declared.

Patient consent for publication Not required.

Ethics approval Ethical approval was obtained from the University of Sydney Human Research Ethics Committee on 29 April 2015 (2014/929) and the Vietnam Ministry of Health Institutional Review Board on 30 September 2015 (4040/ QD-BYT).

Provenance and peer review This is not a commissioned study. The grant application was externally peer-reviewed.

Open access This is an open access article distributed in accordance with the Creative Commons Attribution Non Commercial (CC BY-NC 4.0) license, which permits others to distribute, remix, adapt, build upon this work non-commercially, and license their derivative works on different terms, provided the original work is properly cited, appropriate credit is given, any changes made indicated, and the use is non-commercial. See: http://creativecommons.org/licenses/by-nc/4.0/.

ORCID iD

Greg J Fox http://orcid.org/0000-0002-4085-1411

\section{REFERENCES}

1 World Health Organization. 2018 global tuberculosis report. 2018. Geneva, Switzerland: WHO, 2018.

2 Ahmad N, Ahuja SD, Akkerman OW, et al. Treatment correlates of successful outcomes in pulmonary multidrug-resistant tuberculosis: an individual patient data meta-analysis. Lancet 2018;392:821-34.

3 Knight GM, McQuaid CF, Dodd PJ, et al. Global burden of latent multidrug-resistant tuberculosis: trends and estimates based on mathematical modelling. Lancet Infect Dis 2019;19:903-12.

4 European Centre for Disease Prevention and Control. Management of contacts of MDR TB and XDR TB patients. Stockholm ECDC, 2012.

5 The Stop TB Partnership. The global plan to stop TB 2011-2015. Geneva, 2010.

6 Medecins Sans Frontiers. An evaluation of drug-resistant TB treatment scale-up. Geneva, 2011.

7 World Health Organization. Latent tuberculosis infection. updated and consolidated guidelines for programmatic management. Geneva: WHO, 2018.

8 Menzies D, Adjobimey M, Ruslami R, et al. Four months of rifampin or nine months of isoniazid for latent tuberculosis in adults. $N$ Engl $J$ Med 2018;379:440-53.

9 Sterling TR, Villarino ME, Borisov AS, et al. Three months of rifapentine and isoniazid for latent tuberculosis infection. $N$ Engl J Med 2011;365:2155-66.
10 Byrne AL, Fox GJ, Marais BJ. Better than a pound of cure: preventing the development of multidrug-resistant tuberculosis. Future Microbiol 2018;13:577-88.

11 Fox GJ, Oxlade O, Menzies D. Fluoroquinolone therapy for the prevention of multidrug-resistant tuberculosis in contacts. A costeffectiveness analysis. Am J Respir Crit Care Med 2015;192:229-37.

12 Fox GJ, Schaaf HS, Mandalakas A, et al. Preventing the spread of multidrug-resistant tuberculosis and protecting contacts of infectious cases. Clin Microbiol Infect 2017;23:147-53.

13 Seddon JA, Hesseling AC, Finlayson $\mathrm{H}$, et al. Preventive therapy for child contacts of multidrug-resistant tuberculosis: a prospective cohort study. Clin Infect Dis 2013;57:1676-84.

14 Bamrah S, Brostrom R, Dorina F, et al. Treatment for LTBI in contacts of MDR-TB patients, Federated states of Micronesia, 2009-2012. Int J Tuberc Lung Dis 2014:18:912-8.

15 Marks SM, Mase SR, Morris SB. Systematic review, meta-analysis, and cost-effectiveness of treatment of latent tuberculosis to reduce progression to multidrug-resistant tuberculosis. Clin Infect Dis 2017;64:1670-7.

16 World Health Organization. WHO consolidated guideilnes on drugresistant tuberculosis treatment. Geneva, Switzerland: WHO, 2019.

17 Hurst M, Lamb HM, Scott LJ, et al. Levofloxacin: an updated review of its use in the treatment of bacterial infections. Drugs 2002;62:2127-67.

18 Torre-Cisneros J, San-Juan R, Rosso-Fernández CM, et al. Tuberculosis prophylaxis with levofloxacin in liver transplant patients is associated with a high incidence of tenosynovitis: safety analysis of a multicenter randomized trial. Clin Infect Dis 2015;60:1642-9.

19 Fair E, Miller C, Ottmani S, et al. Tuberculosis contact investigation in low and middle income countries: standardized definitions and indicators. Int J Tuberc Lung Dis. In Press 2015.

20 Second Edition of Management of Multidrug-Resistant Tuberculosis in Children: A Field Guide. Boston: The Sentinel Project for Pediatric Drug Resistant Tuberculosis, 2014.

21 United States Food and Drug Administration (US FDA). Levaquin (levofloxacin) tablets, oral solution and injection, 2014. Available: http://www.fda.gov/safety/medwatch/safetyinformation/ucm303865. htm [Accessed 20 June 2015]

22 Morrison J, Pai M, Hopewell PC. Tuberculosis and latent tuberculosis infection in close contacts of people with pulmonary tuberculosis in low-income and middle-income countries: a systematic review and meta-analysis. Lancet Infect Dis 2008;8:359-68.

23 Fox GJ, Barry SE, Britton WJ, et al. Contact investigation for tuberculosis: a systematic review and meta-analysis. Eur Respir J 2013;41:140-56.

24 Lobue P, Menzies D. Treatment of latent tuberculosis infection: an update. Respirology 2010;15:603-22.

25 Nguyen HB, Nguyen CV, Tran HTG, et al. Prevalence of resistance to second-line tuberculosis drug among multidrug-resistant tuberculosis patients in Viet Nam, 2011. Western Pac Surveill Response J 2016;7:35-40.

26 Fox GJ, Nhung NV, Sy DN, et al. Household-contact investigation for detection of tuberculosis in Vietnam. N Engl J Med 2018;378:221-9.

27 Schlesselman JJ. Sample size requirements in cohort and casecontrol studies of disease. Am J Epidemiol 1974;99:381-4.

28 International Committee of medical Journal editors recommendations: defining the role of authors and contributors. Available: http://www.icmje.org/recommendations/browse/roles-andresponsibilities/defining-the-role-of-authors-and-contributors.html [Accessed 21 Sept 2019].

29 Zenner D, Beer N, Harris RJ, et al. Treatment of latent tuberculosis infection: an updated network meta-analysis. Ann Intern Med 2017;167:248-55. 